KUNS-XXXX

\title{
Classical conformality in the Standard Model from Coleman's theory
}

\author{
Kiyoharu Kawana* \\ Department of Physics, Kyoto University, Kyoto 606-8502, Japan
}

June 2, 2022

\begin{abstract}
The classical conformality is one of the possible candidates for explaining the gauge hierarchy of the Standard Model. We show that it is naturally obtained from the Coleman's theory on baby universe.
\end{abstract}

${ }^{*}$ E-mail: kiyokawa@gauge.scphys.kyoto-u.ac.jp 
Although the Standard Model (SM) is completed by the discovery of the Higgs boson, there are many open questions in it such as the Higgs quadratic divergence, the origin of the electroweak symmetry breaking (EWSB), the cosmological constant problem, and so on. The first two are important in that sense that they are inherent in local field theory even without considering cosmology. Therefore, it is quite important to seek for new theory or mechanism that naturally answer these questions.

The classical conformality (CC) [1] recently attracts much attention as one of the candidates. The basic idea is as follows: The bare Higgs potential is given by

$$
\begin{aligned}
V_{B}\left(h_{B}\right) & =\frac{A_{B}}{2} h_{B}^{2}+\frac{\lambda_{B}}{4} h_{B}^{4} \\
& =\frac{A(\mu)}{2} h^{2}+\frac{\lambda(\mu)}{4} h^{4}+\frac{\delta A(\mu)}{2} h^{2}+\frac{\delta \lambda(\mu)}{4} h^{4},
\end{aligned}
$$

where $\mu$ is the renormalization scale, $A_{B}(A(\mu))$ and $\lambda_{B}(\lambda(\mu))$ are the bare (renormalized) couplings, and $\delta A(\mu)$ and $\delta \lambda(\mu)$ are the counter terms. By using the wave function renormalization $Z_{h}$, they obey

$$
Z_{h} A_{B}=A(\mu)+\delta A(\mu), Z_{h}^{2} \lambda_{B}=\lambda(\mu)+\delta \lambda(\mu) .
$$

As is well known, $\delta A(\mu)$ contains the quadratic divergence, so we must drastically tune the bare mass term $A_{B}$ to obtain $-A(\mu) \sim \mathcal{O}(100 \mathrm{GeV})$. However, such a fine-tuning is automatically taken place if we demand that $A(\mu)$ becomes zero at some scale $\Lambda$ :

$$
0=A(\Lambda)\left(=Z_{h} A_{B}-\delta A(\Lambda)\right) .
$$

Once this is satisfied, the quadratic divergence never appears in the physical quantities . This is the basic idea of the CC. The purpose of this paper is to show that Eq. (4) is naturally concluded from the Coleman's theory. Note that, in the SM, because the renormalization group equation of $A(\mu)$ is proportional to itself, the Higgs mass term never appears if the $\mathrm{CC}$ is realized at some scale. Therefore, other mechanisms such as the $\mathrm{CW}$ mechanism are needed to realize the EWSB, and they are well studied in the context of the models beyond the SM [1]. In such extensions, a few scalars generally exist, and we do not yet understand whether our (following) argument is also applicable to such general cases. In this paper, we just concentrate on the CC in the SM.

The Coleman's theory [2] is one of the promising candidates for solving the naturalness problem, and recently attracts much attention because it is formulated in the Lorentzian framework [3, 4]. It predicts that the parameters (couplings) $\vec{\lambda}$ of local field theory are fixed at the point that strongly dominates in the integration of the partition function of the universe:

$$
Z \sim \int d \vec{\lambda} f(\vec{\lambda}) \exp \left(-i \varepsilon(\vec{\lambda}) V_{4}\right)
$$

where $f(\vec{\lambda})$ is an ordinary function of $\vec{\lambda}, \varepsilon(\vec{\lambda})$ is the vacuum energy density of a system, and $V_{4}$ is the spacetime volume. From this equation, one can easily understand that a special 


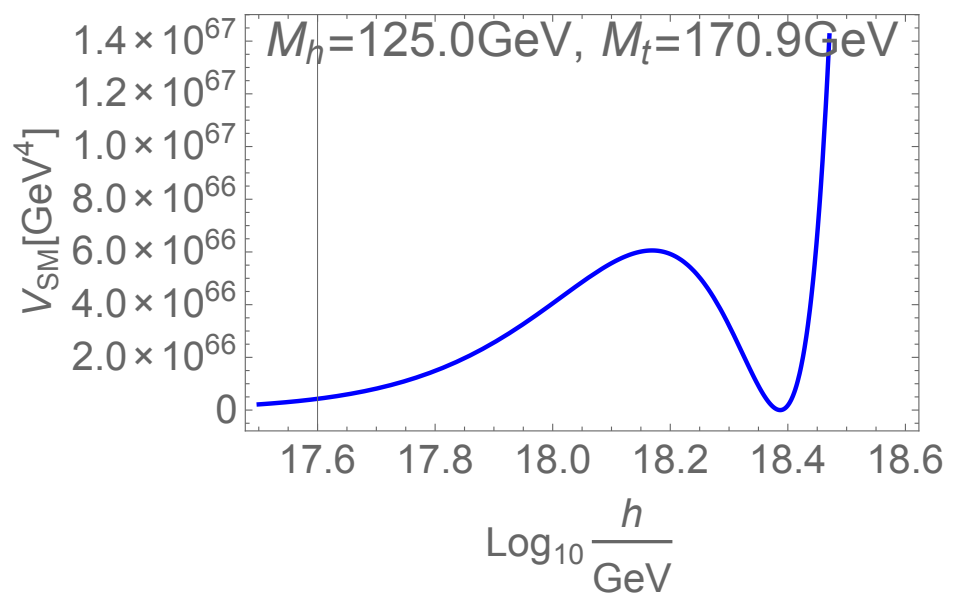

Figure 1: The Higgs potential in the SM. Here, the top mass is fine-tuned so that another vacuum appears around the Planck scale.

point such as a saddle point of $\varepsilon(\vec{\lambda})$ strongly dominates because $V_{4}$ is quite large. See [3, 4] for more details.

In particular, in the recent work [4, it was shown that the Multiple point criticality principle (MPP) [5] can be derived by using the Coleman's theory. The principle is a hypothesis which claims that if there are two vacua in a model, the parameters of the model are fixed so that they become degenerate. Of course, this principle originates from the SM Higgs potential. See Fig.1 for example. This is the Higgs potential of the SM by using the observed Higgs mass $\sim 125 \mathrm{GeV}$, and one can see that there is another degenerate vacuum around the Planck scale.

In the following, we show that the $\mathrm{CC}$ in the SM can be also derived by using Eq.(5). We first review the result in 4$]$ because the MPP plays an important role for the following discussion.

Let us first consider a general potential $V(\phi, \lambda)$ of a scalar field $\phi$ having two minima at $\phi_{1}(\lambda)$ and $\phi_{2}(\lambda)$, where we take $\phi_{1}(\lambda)<\phi_{2}(\lambda)$. Here, $\lambda$ is one of the coupling constants of the theory. For example, it corresponds to the Higgs quartic coupling or the top Yukawa in the SM. We assume that two minima become degenerate when $\lambda$ is equal to zero, and that the signature of $\lambda$ is chosen as

$$
\begin{array}{ll}
V\left(\phi_{1}(\lambda), \lambda\right)<V\left(\phi_{2}(\lambda), \lambda\right) & \text { for } \lambda>0, \\
V\left(\phi_{1}(\lambda), \lambda\right)>V\left(\phi_{2}(\lambda), \lambda\right) & \text { for } \lambda<0 .
\end{array}
$$

See Fig, 2 for example. Then, the true vacuum expectation value $\phi_{\text {vac }}(\lambda)$ and the vacuum 


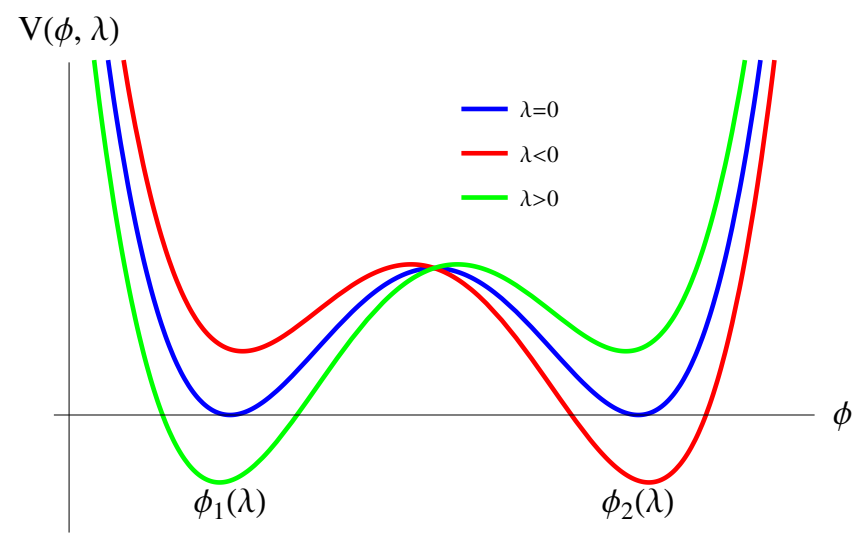

Figure 2: Schematic behavior of $V(\phi, \lambda)$. The blue line corresponds to $\lambda=0$, and the green (red) line corresponds to $\lambda>0(<0)$.

energy density $\varepsilon(\lambda)$ are given by

$$
\phi_{\mathrm{vac}}(\lambda)=\left\{\begin{array}{ll}
\phi_{2}(\lambda) & \text { for } \lambda<0 \\
\phi_{1}(\lambda) & \text { for } \lambda>0
\end{array} \quad, \quad \varepsilon(\lambda)= \begin{cases}V\left(\phi_{2}(\lambda)\right) & \text { for } \lambda<0 \\
V\left(\phi_{1}(\lambda)\right) & \text { for } \lambda>0 .\end{cases}\right.
$$

Now, in order to examine the dominant point in Eq.(5), let us use the following mathematical formula: If $g(\lambda)$ is smooth and monotonic in the $\lambda>0$ region, and $g^{\prime}(0) \neq 0$, we have

$$
e^{i k g(\lambda)} \theta(\lambda) \underset{k \rightarrow \infty}{\sim} \frac{i}{k}\left(\frac{d g}{d \lambda}\right)^{-1} e^{i k g(0)} \delta(\lambda)
$$

where $\theta(\lambda)$ is a step function. The proof is as follows. By multiplying a test function $F(\lambda)$ with finite support to $e^{i k g(\lambda)}$, and integrating from 0 to $\infty$, we obtain

$$
\begin{aligned}
\int_{0}^{\infty} d \lambda e^{i g(\lambda) k} F(\lambda) & =\int_{g(0)}^{\infty} d g\left(\frac{d g}{d \lambda}\right)^{-1} e^{i k g} F(\lambda=\lambda(g)) \\
& =\left[\frac{e^{i k g}}{i k}\left(\frac{d g}{d \lambda}\right)^{-1} F(\lambda(g))\right]_{g(0)}^{\infty}+\mathcal{O}\left(\frac{1}{k^{2}}\right) \\
& =\left.\frac{i}{k}\left(\frac{d g}{d \lambda}\right)^{-1} e^{i k g(0)} F(\lambda)\right|_{\lambda=0} ^{+\mathcal{O}\left(\frac{1}{k^{2}}\right) .}
\end{aligned}
$$

Thus, Eq. (8) holds in the $k \rightarrow \infty$ limit.

By assuming that $V\left(\phi_{1}(\lambda)\right)$ and $V\left(\phi_{2}(\lambda)\right)$ are monotonic in $\lambda>0$ and $\lambda<0$ respectively, and using Eq. (8), we have

$$
e^{-i \varepsilon(\lambda) V_{4}} \sim-\frac{i e^{-i \varepsilon(0) V_{4}}}{V_{4}} \times\left[\left.\left(\frac{V\left(\phi_{1}(\lambda)\right)}{d \lambda}\right)^{-1}\right|_{0+}-\left.\left(\frac{V\left(\phi_{2}(\lambda)\right)}{d \lambda}\right)^{-1}\right|_{0-}\right] \delta(\lambda),
$$



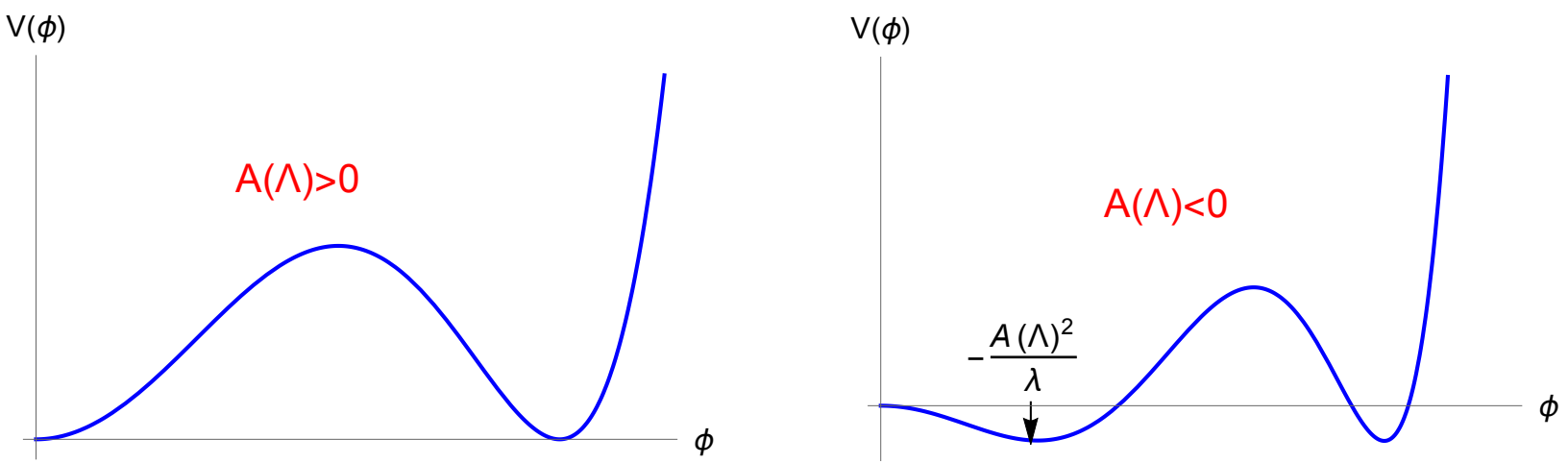

Figure 3: The typical behaviors of the Higgs potential where the MPP is realized. Left (Right) panel shows the $A(\Lambda)>0(<0)$ case.

which leads to

$$
Z \sim \frac{f(0)}{V_{4}} \times e^{-i \varepsilon(0) V_{4}} .
$$

This is the derivation of the MPP in the context of the Coleman's theory. In the SM case, the Higgs potential can have actually two degenerate vacua with vanishing vacuum energy. (See again Fig, 1.)

Let us now derive the $\mathrm{CC}$ by assuming that the MPP is always realized from the above argument. In the $\mathrm{SM}$, because $A(\mu)$ is proportional to $A(\Lambda)$, we treat the latter as a free parameter in the following. In this case, the vacuum energy density is given by

$$
\varepsilon(A(\Lambda))=\left\{\begin{array}{ll}
0 & \text { for } A(\Lambda)>0 \\
-\frac{A(\Lambda)^{2}}{\lambda} & \text { for } A(\Lambda)<0
\end{array},\right.
$$

where $\lambda$ is the effective Higgs quartic coupling at the weak scale $\sim \mathcal{O}(0.1)$. See Fig 3 for example. Therefore, by using Eq.(8), we can see that $A(\Lambda)=0$ dominates at least in the $A(\Lambda)<0$ region, wheres there seems no dominant point in the $A(\Lambda)>0$ region. However, in addition to the above vacuum energy, there is always a contribution from the zero point oscillation energy:

$$
\varepsilon_{\text {zero }}(A(\Lambda))=\int \frac{d^{3} \mathbf{k}}{(2 \pi)^{3}} \sqrt{\mathbf{k}^{2}+c A(\Lambda)}
$$

where $c$ is a positive $\mathcal{O}(1)$ coefficient determined by the RGE of the SM. This is apparently a monotonic function of $\Lambda(\Lambda)$, so we can conclude that $A(\Lambda)=0$ also dominates even in the $A(\Lambda)>0$ region 1 . This is the derivation of the CC in the SM. Although we have not

\footnotetext{
${ }^{1}$ The zero point energy also exists in the $A(\Lambda)<0$ region. However, this does not change our conclusion because it is also monotonic.
} 
considered the extensions of the SM here, it might be interesting to study whether the CC is also realizable even in such extensions while preserving their phenomenological aspects such as the EWSB.

\section{Acknowledgement}

This work is supported by the Grant-in-Aid for Japan Society for the Promotion of Science (JSPS) Fellows No.27·1771.

\section{References}

[1] K. A. Meissner and H. Nicolai, "Conformal Symmetry and the Standard Model," Phys. Lett. B 648, 312 (2007) doi:10.1016/j.physletb.2007.03.023 hep-th/0612165; R. Foot, A. Kobakhidze, K. L. McDonald and R. R. Volkas, "A Solution to the hierarchy problem from an almost decoupled hidden sector within a classically scale invariant theory," Phys. Rev. D 77, 035006 (2008) doi:10.1103/PhysRevD.77.035006 arXiv:0709.2750 [hep-ph]]; K. A. Meissner and H. Nicolai, "Effective action, conformal anomaly and the issue of quadratic divergences," Phys. Lett. B 660, 260 (2008) doi:10.1016/j.physletb.2007.12.035 [arXiv:0710.2840 [hep-th]]; S. Iso, N. Okada and Y. Orikasa, "Classically conformal $B^{-}$L extended Standard Model," Phys. Lett. B 676, 81 (2009) doi:10.1016/j.physletb.2009.04.046 arXiv:0902.4050 [hepph]]; S. Iso, N. Okada and Y. Orikasa, "The minimal B-L model naturally realized at TeV scale," Phys. Rev. D 80, 115007 (2009) doi:10.1103/PhysRevD.80.115007 arXiv:0909.0128 [hep-ph]]. L. Alexander-Nunneley and A. Pilaftsis, "The Minimal Scale Invariant Extension of the Standard Model," JHEP 1009, 021 (2010) doi:10.1007/JHEP09(2010)021 arXiv:1006.5916 [hep-ph]]; S. Iso and Y. Orikasa, "TeV Scale B-L model with a flat Higgs potential at the Planck scale - in view of the hierarchy problem -," PTEP 2013, 023B08 (2013) doi:10.1093/ptep/pts099 arXiv:1210.2848 [hep-ph]]; R. Foot, A. Kobakhidze and A. Spencer-Smith, "Criticality in the scale invariant standard model (squared)," Phys. Lett. B 747, 169 (2015) doi:10.1016/j.physletb.2015.05.064 arXiv:1409.4915 [hep-ph]]; H. Okada and Y. Orikasa, "Classically Conformal Radiative Neutrino Model with Gauged B-L Symmetry," arXiv:1412.3616 [hep-ph]; K. Kawana, PTEP 2015, 073B04 (2015) doi:10.1093/ptep/ptv093 arXiv:1501.04482 [hep-ph]]; N. Haba and Y. Yamaguchi, "Vacuum stability in the $U(1)_{\chi}$ extended model with vanishing scalar potential at the Planck scale," PTEP 2015, no. 9, 093B05 (2015) doi:10.1093/ptep/ptv121 arXiv:1504.05669 [hep-ph]]; N. Haba, H. Ishida, R. Takahashi and Y. Yamaguchi, "Gauge coupling unification in a classically scale invariant model," JHEP 1602, 058 (2016) doi:10.1007/JHEP02(2016)058 arXiv:1511.02107 [hep-ph]]; N. Haba, 
H. Ishida, N. Kitazawa and Y. Yamaguchi, "A new dynamics of electroweak symmetry breaking with classically scale invariance," Phys. Lett. B 755, 439 (2016) doi:10.1016/j.physletb.2016.02.052 [arXiv:1512.05061 [hep-ph]].

[2] S. R. Coleman, "Why There Is Nothing Rather Than Something: A Theory of the Cosmological Constant," Nucl. Phys. B 310, 643 (1988). doi:10.1016/05503213(88)90097-1

[3] H. Kawai and T. Okada, Prog. Theor. Phys. 127, 689 (2012) doi:10.1143/PTP.127.689 arXiv:1110.2303 [hep-th]]; Y. Hamada, H. Kawai and K. Kawana, "Evidence of the Big Fix," Int. J. Mod. Phys. A 29, 1450099 (2014) doi:10.1142/S0217751X14500997 arXiv:1405.1310 [hep-ph]]; Y. Hamada, H. Kawai and K. Kawana, "Weak Scale From the Maximum Entropy Principle," PTEP 2015, 033 B06 (2015) doi:10.1093/ptep/ptv011 arXiv:1409.6508 [hep-ph]].

[4] Y. Hamada, H. Kawai and K. Kawana, "Natural solution to the naturalness problem: The universe does fine-tuning," PTEP 2015, no. 12, 123B03 (2015) doi:10.1093/ptep/ptv168 [arXiv:1509.05955 [hep-th]].

[5] C. D. Froggatt and H. B. Nielsen, "Standard model criticality prediction: Top mass 173 +- 5-GeV and Higgs mass 135 +- 9-GeV," Phys. Lett. B 368, 96 (1996) doi:10.1016/0370-2693(95)01480-2 hep-ph/9511371]; C. D. Froggatt, H. B. Nielsen and Y. Takanishi, "Standard model Higgs boson mass from borderline metastability of the vacuum," Phys. Rev. D 64, 113014 (2001) doi:10.1103/PhysRevD.64.113014 hep-ph/0104161]; K. Kawana, "Multiple Point Principle of the Standard Model with Scalar Singlet Dark Matter and Right Handed Neutrinos," PTEP 2015, 023B04 (2015) doi:10.1093/ptep/ptv006 arXiv:1411.2097 [hep-ph]]; Y. Hamada and K. Kawana, "Vanishing Higgs Potential in Minimal Dark Matter Models," Phys. Lett. B 751, 164 (2015) doi:10.1016/j.physletb.2015.10.006 [arXiv:1506.06553 [hep-ph]]. 